\title{
Participants' Explanatory Model of Being Overweight and Their Experiences of 2 Weight Loss Interventions
}

\author{
Amy L. Abern, $P b D^{1}$ \\ Emma J. Boyland, $P b D^{1}$ \\ Susan A. Jebb, $P b D^{1}$ \\ Simon R. Cobn, $P b D^{2}$ \\ 'MRC Human Nutrition Research, \\ Cambridge, United Kingdom
}

${ }^{2}$ Institute of Public Health, University of Cambridge, Cambridge, United Kingdom

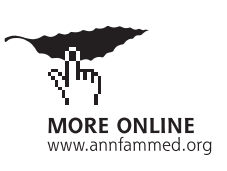

Conflicts of interest: Drs Abern and Jebb have received funding to their institution from Weight Watchers International for the trial described in this report. Dr Jebb has received research grants for other clinical trials from Sanofi-Aventis and Coca Cola she is also a member of the Tanita Medical Advisory Board and has received payment for nutrition articles and lectures for Rosemary Conley Enterprises.

\section{CORRESPONDING AUTHOR}

Amy L. Ahern, PhD

MRC Human Nutrition Research

Elsie Widdowson Laboratory

Fulbourn Rd

Cambridge, CB1 9NL, UK

Amy.Ahern@mrc-hnr.cam.ac.uk

\begin{abstract}
PURPOSE We explored participants' accounts of weight loss interventions to illuminate the reasons behind the greater weight loss observed among those attending a commercial program compared with those receiving standard care in a recent large-scale trial. We further wanted to examine how participants' general explanatory model of being overweight related to the 2 different interventions.
\end{abstract}

METHODS Our study was based on thematic analysis of semistructured telephone interviews with a purposeful sample of 16 female participants from the UK center of a randomized controlled trial of weight loss in primary care.

RESULTS The commercial provider delivered weight management in a nonmedical context, which mirrors how participants regard being overweight. Participants felt they needed support and motivation rather than education, and valued the ease of access and frequent contact the commercial provider offered. Some participants preferred individual level support with their primary care clinician, and all were positive about the opportunity to access support through the primary care setting.

CONCLUSIONS Primary care referral to a commercial weight loss program for people who do not require specific clinical care appears to be in accord with their general explanatory model about being overweight, offering motivation and support to lose weight outside a strictly medical context. This approach may not be effective or acceptable for everyone, however, and there are likely to be considerable variations in the explanatory models held. Findings support the argument that a range of evidence-based options for weight management should be available in primary care.

Ann Fam Med 2013;11:251-257. doi:10.1370/afm.1446.

\section{INTRODUCTION}

It is well established that obesity is associated with considerable health consequences, including diabetes, cardiovascular disease, and some cancers. ${ }^{1}$ Intensive lifestyle interventions led by health professionals can produce clinically significant weight loss of $5 \%$ to $10 \%,{ }^{2}$ but such interventions are costly given the high prevalence of obesity. Nevertheless, obesity accounts for $2 \%$ to $7 \%$ of health care costs in some developed countries, ${ }^{1}$ so governments are increasingly making prevention and treatment of obesity a priority. For example, the US Center for Medicare and Medicaid Services now includes intensive behavioral counseling for obesity in its coverage, providing it is delivered by a primary care physician in a primary care setting. ${ }^{3}$ Even so, interventions delivered in primary care can be costly in terms of staff resources, setup, and training, and weight loss achieved is often less than $5 \%$ of initial weight. ${ }^{4-6}$ In the United Kingdom, the National Institute for Health and Clinical Excellence recommends consideration of any intervention that meets best practice guidelines, including referral to com- 
mercial weight loss programs ${ }^{7}$ that are delivered to large groups and are thus more affordable. Audit data show the increasing use of referral schemes in the United Kingdom, ${ }^{8,9}$ and 2 recent randomized controlled trials provide evidence to support this approach. ${ }^{10,11}$

We recently published the results of an international randomized controlled trial in which 772 participants from 3 countries (United Kingdom, Australia, and Germany) were recruited by their primary care physician and randomized to receive 12 months' free membership of a commercial program, eg, Weight Watchers, or standard care in general practice. ${ }^{10}$ Those allocated to a commercial program lost twice as much weight as those who received standard care and were 3 times as likely to lose $5 \%$ or more of their initial weight. Full details of this trial are reported elsewhere. ${ }^{10}$

At the end of the trial, we interviewed a sample of UK participants and used qualitative methods to explore accounts of their experience of the 2 interventions, as well as their previous experience of weight management. Our overarching approach was to capture the general explanatory model that all participants held about being overweight and to examine participants' experience of the weight loss interventions within this context. It also considered how patients regard the roles of primary care clinicians and their attitudes toward partnerships with commercial providers.

\section{METHODS}

\section{Sampling and Data Collection}

Sixteen female participants were recruited from the UK center of a trial comparing primary care referral to a commercial weight loss program (Weight Watchers) with standard care. ${ }^{10}$ The main aspects of the 2 interventions are detailed in Table 1 . The participants were purposefully sampled to represent both intervention groups according to basic descriptive variables and to ensure we had respondents from each participating practice, completers and noncompleters, and different levels of weight loss to provide maximum potential variation in accounts.

Participants completed a semistructured telephone interview with one author (A.L.A.) within 6 months of their 12-month assessment date (see the Supplemental Appendix at http://www.

annfammed.org/content/11/3/251/

HC1). The interview schedule was developed after a review of the literature and consideration of topics raised by participants and practitioners during firsthand interactions throughout the main trial. Specific prompts, such as, "What were your expectations of treatment?" "What, if any, do you think are your main barriers to losing weight?" and "How do you feel about your weight now?" were embedded in a narrative-style interview approach that encouraged participants not only to give an account of their experiences chronologically but also to elaborate their general views and beliefs. Any previous experience of weight loss initiatives in primary care, both within and outside the trial, was also elicited.

Written informed consent, including consent to have their interview recorded and transcribed, was obtained from all participants and reviewed verbally immediately before each interview. This study was approved by Nottingham Research Ethics Committee, United Kingdom.

\section{Data Analysis}

Interviews were digitally recorded and transcribed verbatim. An iterative thematic analysis was conducted according to an initial and relatively open interpretive framework derived from the topic guide. ${ }^{12}$ All of the transcripts were read by 3 researchers (A.L.A., E.J.B., S.R.C.), who identified main themes and ideas independently and then met together to reach consensus and establish reliability. Two of the researchers (A.L.A., E.J.B.) worked together to augment the original themes and identify key areas that in combination constituted a general model of beliefs and values associated with being overweight. A final set of themes was established once no new ones emerged from the data set; in contrast to an entirely open grounded theory approach, data satura-

\begin{tabular}{|ll}
\hline Table 1. Characteristics of the Weight Loss Interventions \\
\hline Intervention & Characteristics \\
\hline $\begin{array}{c}\text { Commercial } \\
\text { program }\end{array}$ & Vouchers to attend Weight Watchers for 12 months \\
& Weekly group meetings in local community venue \\
& Promotes a hypoenergetic, balanced diet based on healthy eating \\
principles & \\
& Advice on increasing physical activity \\
& Weight measurement \\
& Group support \\
& Access to internet-based systems to monitor food intake, activity, and \\
& weight change; to participate in community discussion boards; and \\
& to access a library of information, recipes, and meal ideas \\
Average participant attendance while in trial = 3 meetings per month & In line with national guidelines (see http://www.nice.org.uk/CG043) \\
Weight loss advice from primary care professional at local practice \\
(usually practice nurse) \\
1-on-1 meetings; minimum level of care = 6 visits over 12 months \\
Weight measurement \\
Dietary advice based on British Heart Foundation booklet So You \\
Want To Lose Weight... For Good \\
Average participant attendance while in trial = 1 meeting per month
\end{tabular}


Table 2. Characteristics of Study Participants, and UK Trial Participants From Which They Are Drawn

\begin{tabular}{lcccccc}
\hline & \multicolumn{2}{c}{ Commercial Program } & \multicolumn{2}{c}{ Standard Care } & \multicolumn{2}{c}{ Overall } \\
\cline { 2 - 7 } Characteristic & $\begin{array}{c}\text { UK RCT } \\
\text { Participants } \\
(\mathbf{n}=\mathbf{1 2 0})\end{array}$ & $\begin{array}{c}\text { Interview } \\
\text { Sample } \\
(\mathbf{n}=\mathbf{9})\end{array}$ & $\begin{array}{c}\text { UK RCT } \\
\text { Participants } \\
(\mathbf{n}=\mathbf{1 1 6})\end{array}$ & $\begin{array}{c}\text { Interview } \\
\text { Sample } \\
(\mathbf{n}=\mathbf{7})\end{array}$ & $\begin{array}{c}\text { UK RCT } \\
\text { Participants } \\
(\mathbf{n}=\mathbf{2 3 6})\end{array}$ & $\begin{array}{c}\text { Interview } \\
\text { Sample } \\
(\mathbf{n}=\mathbf{1 6})\end{array}$ \\
\hline Age, mean y & 47 & 44 & 46 & 49 & 47 & 47 \\
Entry BMI, mean kg/m² & 31 & 30 & 31 & 31 & 31 & 31 \\
Ethnicity, \% white & 95 & 100 & 95 & 89 & 95 & 94 \\
Female, \% & 92 & 100 & 91 & 100 & 91 & 100 \\
\hline BMI = body mass index; RCT = randomized controlled trial; UK = United Kingdom. &
\end{tabular}

tion was consequently achieved by the predetermined limits of our initial topic guide. One researcher (E.J.B.) continually recoded all transcripts where necessary, collating sections of data that supported and refuted each theme for review by the rest of the research team.

The overall rationale of the interview schedule was to elicit the general views of participants about being overweight, both in relation to themselves and others. We sought to establish what key themes constituted a general cultural explanatory model of being overweight and the extent to which this model framed how they described their experiences in the trial. We use the term explanatory model to encapsulate the ideas about a particular health issue that are intrinsically related to beliefs about its status as an illness, what strategies are believed to be effective, and who are considered the most appropriate people to help. ${ }^{13}$

In adopting this approach, we explicitly avoided using the descriptors patient and treatment, because such terms imply a dominant medical model and do not necessarily reflect participants' own beliefs about being overweight. Although explanatory models by definition vary among different people, in this study, we sought only to establish the general characteristics across the trial cohort via a representative sample of participants. The emerging themes were consequently grouped together with this purpose in mind and assembled into a general hierarchy to establish the overall dominant themes.

\section{RESULTS}

The basic descriptive variables of the intervention groups are displayed in Table 2. A description of the individual participants is displayed in Table 3. Key aspects of the participants' cultural explana- tory model are outlined in Table 4 , with descriptions of how the experiences of the 2 interventions fit with the explanatory model.

\section{Background Accounts: \\ Experiences Before the Trial}

Participants reported prior experience of weight loss and weight regain but had little experience of assistance with weight loss from primary care clinicians. A small number had been told by their general practitioner that their weight was a health issue, but none had been offered any support with losing weight. Very few had specifically sought support from their physician for weight management, and most were unaware it was available. On the whole, participants described weight loss as a personal responsibility and not important enough to bother their general practitioner. Despite having a body mass index of greater than $30 \mathrm{~kg} / \mathrm{m}^{2}$, none considered themselves to be obese, instead describing themselves as overweight or too fat. Participants did not think of their

\begin{tabular}{|cccccc|}
\hline \multicolumn{5}{|c}{ Table 3. Characteristics of the Individual Participants } \\
\hline Participant & Intervention & Completed & $\begin{array}{c}\text { Time in } \\
\text { Trial, mo }\end{array}$ & $\begin{array}{c}\text { Baseline } \\
\text { Weight } \\
\text { (kg) }\end{array}$ & $\begin{array}{c}\text { Weight } \\
\text { Change, } \\
\%\end{array}$ \\
\hline 1 & SC & Withdrew & 4 & 81.0 & 1.98 \\
2 & SC & Completed & 12 & 70.0 & -7.43 \\
3 & CP & Completed & 12 & 81.7 & -17.87 \\
4 & CP & Withdrew & 4 & 80.0 & -3.5 \\
5 & SC & Completed & 12 & 64.8 & -0.77 \\
6 & CP & Completed & 12 & 81.8 & -13.57 \\
7 & SC & Completed & 12 & 87.5 & -13.71 \\
8 & CP & Withdrew & 4 & 71.3 & 0.14 \\
9 & CP & Completed & 12 & 86.8 & -11.29 \\
10 & SC & Withdrew & Baseline only & 90.7 & - \\
11 & SC & Completed & 12 & 73.8 & -9.08 \\
12 & SC & Withdrew & 9 & 74.6 & -0.94 \\
13 & SC & Withdrew & Baseline only & 74.9 & - \\
14 & SC & Withdrew & 2 & 76.8 & -5.6 \\
15 & CP & Withdrew & 2 & 87.2 & -1.95 \\
16 & CP & Withdrew & 9 & 88.6 & -0.68 \\
\hline CP $=$ commercial provider; SC standard care. & & & \\
\hline
\end{tabular}




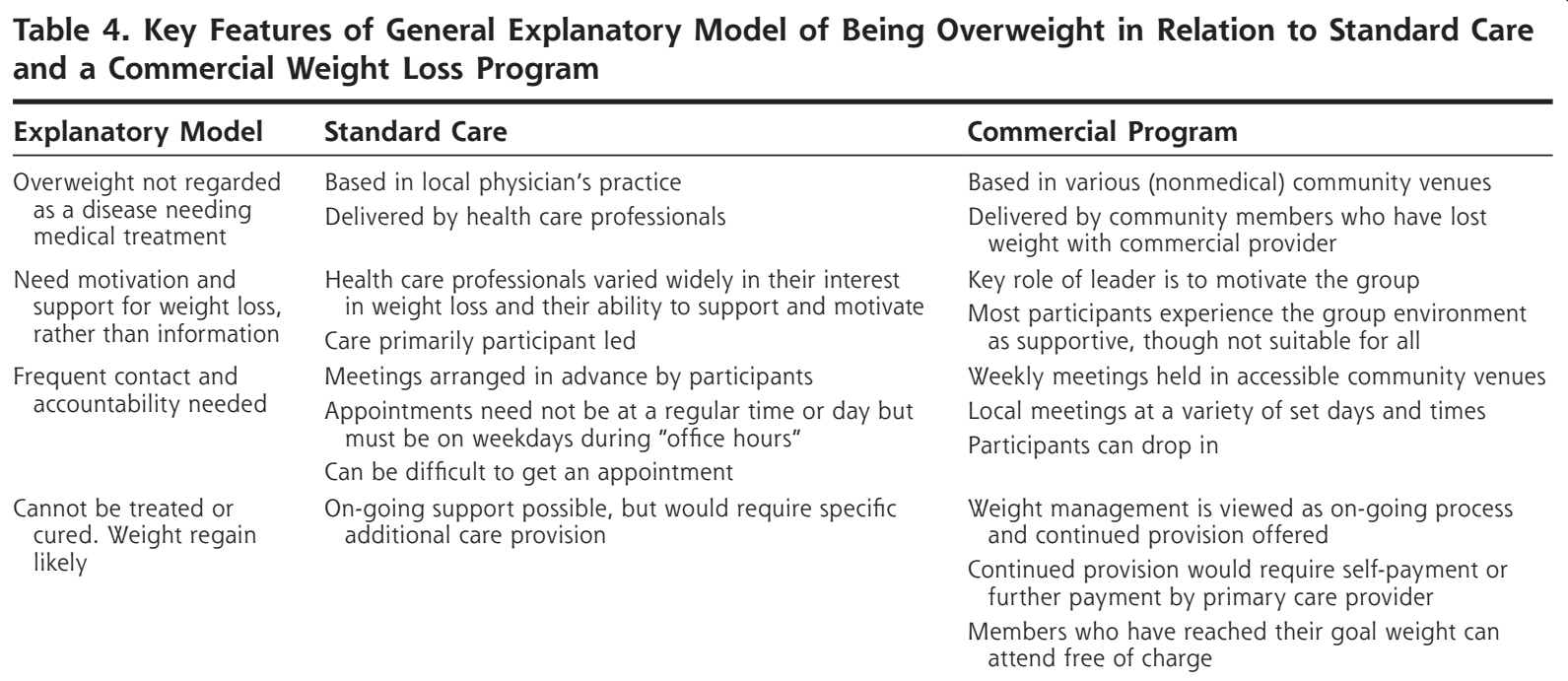

weight as a medical problem, although they perceived obesity as being a medical issue with associated health implications relevant to primary care:

No, not really, no, I wouldn't go to the doctors for weight loss (Participant 1).

Why not (A.L.A.)?

I don't know. Unless there was something medically wrong with me, I wouldn't think it was one of their problems. Because it's your lifestyle, isn't it? It's how you eat and move around or not (Participant 1).

In contrast, 13 participants (81\%) had previous experience of attending commercial weight loss programs. They generally described the experience as positive and defined it as successful if weight was lost while attending, even though weight had been regained afterward in all cases. All participants were familiar with leading commercial providers and typically expressed trust in the brand names. Some skepticism was expressed on their profit-making nature, with suggestions that there was not adequate support for weight maintenance because members who maintain their goal weight do not pay to attend. Even so, the great majority of participants believed that if a commercial program had a proven track record and their approach was perceived as healthy, it provided an appropriate setting for weight loss support.

\section{Descriptions of Weight Loss Provision During the Trial}

Contact and Structure

Participants emphasized the importance of regular contact in order to maintain motivation and focus on the weight loss goals, although there was significant variation in what was perceived as the ideal frequency.
I need to go regularly to keep me on track (Participant 1).

I went every couple of weeks...they would have liked me to have gone every week but...that doesn't suit me (Participant 3).

Greater frequency of contact was seen as a benefit of the commercial program compared with standard care and was associated with other organizational features of the 2 weight loss approaches. The commercial program was largely perceived as structured, with weekly meetings at set times. Some participants believed that structure was incompatible with their busy routines:

"With the Weight Watchers you can only go to a class when it's on... I don't have a regular shift pattern" (Participant 15). Others, however, acknowledged there was always a meeting available if they chose to be adaptable about which group to attend, and not having to make an appointment was seen by some as adding to the flexibility and ease of participation.

Conversely, the appointment-based format in primary care was referred to as ad hoc and experienced as predominantly participant led. Though some appreciated this arrangement, many expressed frustration about limited appointment availability. Appointments generally had to be initiated by participants, and access problems sometimes encountered at their general practitioner's office meant they felt they had to create their own support. Following our theoretical approach, participants' explanatory model appears to suggest weight loss interventions should balance the need to provide a sense of agency while not making the individual entirely responsible for their weight management.

\section{A Sense of Support and Accountability}

Perhaps counter to public health assumptions, none of the participants talked about needing an intervention to include education about food, eating, or diet, as they 
believed they already had the necessary knowledge. Instead, they referred to the importance of receiving motivation and support, although some found it difficult to specify what form the support should take:

It isn't that I need educating, it's more that I need motivating (Participant 1).

I just think I couldn't do it on my own without seeing somebody (Participant 5).

Related to such comments was a general sense of what we have chosen to call accountability. Accountability was engendered by attending either type of session, especially through the act of being weighed rather than weighing themselves. It was identified by many as the key motivating factor for successful weight loss, accompanying a sense of obligation and that they would be "letting someone else down" if they had not lost weight: "For me... what works is the fact that I know...I've got to go and see somebody... and I've got to explain why I haven't lost any weight" (Participant 6).

The related themes of support and accountability underscored the largely positive accounts of the commercial program, in which it was reported that even though the program was group based, it provided good, individually tailored advice. Several commented specifically on the positive, encouraging, and supportive approach of the commercial program generally and of the group leader in particular: "They congratulated you as much for losing half a pound than they would if you lost half a stone" (Participant 9).

In addition, the group format was deemed to create an atmosphere of collective motivation, an opportunity to share experiences, allow talk to focus on problematic behaviors raised by members, and provide a source of inspiration derived from the success of other members. Crucially, the sense of support and accountability was driven not by the fear of embarrassment that might be associated with peer pressure, but by the feelings of loyalty and obligation to the program leader and the group members: "That class motivation I felt worked... building up that... friendly atmosphere and team motivation I found worked quite well" (Participant 12).

Some participants nevertheless felt that group leaders were inclined to apportion blame to a member if there had been no weight loss, and there was insufficient acknowledgment of weight maintenance as a valid, complementary aim.

Some standard care participants described how the opportunity to be weighed in private at the physician's office was preferable and more supportive: "Just doing it on an individual basis meant I could...be more private about it...without having to...go and be weighed in front of everybody" (Participant 11).
These standard care participants talked about commercial programs in terms of peer pressure and the use of stigma as a crude source of motivation. The group weigh-in aspect of the commercial programs was particularly highlighted as a likely source of embarrassment that might deter those with more extreme weight problems from attending. One standard care participant described the commercial program as a "social pressure group."

The summaries of the standard care sessions suggested that there was considerable variation between physician practices in terms of content and delivery. Several reported the style was relatively passive, which, from the participants' point of view, reflected the low priority it was given. Some said the advice given was no better than that found on relevant Web sites or said that their time could be better spent going to the gym. Although some believed they had to rely on themselves to provide too much of the initiative, others interpreted this attribute as positive, which fitted the type of support they wanted:

I just don't think that [support with weight management] seemed to be of particular importance to them (Participant 12).

It was more of a personal journey with medical support.... It was just how I wanted it (Participant 14).

\section{Making Sense of Personal Results}

Whatever their results after the trial, participants largely attributed their success or failure to lose weight to the allocation they were given through randomization. This retrospective rationalization drew on a repertoire around perceived differences in levels of encouragement, a sense of inspiration derived from monitoring, and notions of support and accountability:

If I'd gone to Weight Watchers and had to go every week and I got somebody monitoring me...I feel that that would have really, really encouraged me to do it (Participant 10).

Weight Watchers was a structured plan and the GP [general practitioner] was more trial and error yourself really, but I actually think the GP worked better (Participant 5).

I don't think that I would have been inspired enough really [on the standard care arm] (Participant 6).

Although it may well be that some people would have responded better to the style of assistance offered in the other trial arm, it seems just as likely that attribution of outcome would always have followed this pattern.

In contrast, some participants reported that the treatment arm they were allocated to simply "didn't work for them." This apparently innocuous explanation implies a belief that different kinds of people are suited to different kinds of support. Thus, in terms of the participants' 
explanatory model, being overweight is not conceived of as the same problem for all people but is a very personal issue; as a consequence, weight loss efforts call for a meaningful match between the kind of help given and how a person makes sense of trying to lose weight.

\section{Commercial Partnerships and Use of Health Service Resources}

The commercial program was clearly positioned as a nonmedical intervention. Even so, participants did not report any concerns about group leaders not having professional qualifications. Indeed, a nonmedical approach was seen as an appropriate context in which to receive weight loss support. The potential availability of weight loss assistance paid for through the National Health Service (NHS) was seen as an attractive alternative to having to pay for the commercial treatment. Some described a sense of obligation because attendance was being paid for them by the NHS: "I had to use my little voucher every week (Participant 9).

Others felt less pressure to lose weight because they were not personally contributing financially to attend: "It almost felt because it was being funded, I didn't have the pressure there.... I felt more comfortable with it" (Participant 3).

Most interviewees were initially hesitant about whether weight loss was a legitimate focus for their general practitioner and were concerned that it might constitute a waste of NHS resources. By the end of the trial, however, many standard care participants felt that the experience had changed their views, and that they were now more likely to approach their physician for help in the future. It should be noted though that in the great majority of cases, a nurse or health care assistant was responsible for providing support during the trial, which was regarded as more appropriate for weight management than seeing a physician. Interestingly, although participants who had received standard care during the trial perceived this support as in addition to what they might normally receive, they did not attribute monetary value to it in any way.

\section{DISCUSSION}

This study is based on a sample of UK participants from a randomized controlled trial of weight loss in primary care, which found that a commercial provider was more successful than standard care in helping participants lose weight. ${ }^{10}$ Though limited to a small representative sample from only one of the participating countries, our study enhances the main findings by exploring participants' general views and beliefs about being overweight and the ways these views relate to experiences of the 2 interventions. Our findings sug- gest that by providing weight loss support outside a medical context, referral to a commercial provider resonates with a general explanatory model of being overweight. Included are notions relating to responsibility and agency, ideas of what is experienced as effective and supportive, feelings of accountability and obligation with those charged with helping them, and a resistance to the medicalization of being overweight.

Although data are limited on the experience of weight loss interventions in primary care from the participant's perspective, our findings are consistent with cross-sectional data indicating that professional credentials are not important to patients ${ }^{14}$ and that patients are reluctant to approach their doctors about weight concerns. ${ }^{15}$ Our findings strengthen recent evidence of the clinical effectiveness of commercial weight loss providers ${ }^{10,11}$ and inform the consideration of wider rollout of such service provision. Together they support an approach being adopted in the United Kingdom of including commercial partnerships as an option for weight management in primary care and have implications for health service provision in other countries. Even so, commercial provision may not be suitable for all people wanting to lose weight, and some preferred an individual approach. These differences highlight the need to offer people a range of different evidence-based options and to consider what best suits their needs and lifestyle.

In addition, there was some skepticism around weight maintenance, although to a lesser degree than in some previous research. ${ }^{16}$ Although the trial upon which our study is based examined weight loss over a period of 12 months (considered by the National Institute for Health and Clinical Excellence as a long-term outcome), ${ }_{1}^{7}$ posttreatment weight regain is common in obesity, ${ }^{2}$ a problem not restricted to commercial programs. Participants' general explanatory model, in which being overweight is linked to ongoing experiences of everyday life, suggests that it may be relevant to consider weight management as an on-going process and to focus on sustainability.

A limitation of the qualitative approach is that generation of data is subject to layers of social construction. ${ }^{11}$ Although some studies have found the quality of telephone interviews to be comparable to face-to-face interviews, ${ }^{17}$ it is possible that this method of data collection will have influenced and may have restricted responses. That the interviews were often extensive and the data proved so illuminating, however, suggests the interviewer successfully ensured the participants came to feel at ease and talk openly. It might also be that the greater anonymity elicited more open responses. Particular care was taken to ensure participants' comments were interpreted in the wider context in which they 
were spoken, and every effort was given to ensuring a fair representation of the original intended meaning. Although interviews could only address participant perspectives retrospectively, this limitation was also carefully considered throughout the analysis.

The key themes of the explanatory model that we have drawn on, while useful to address our main research question, are necessarily broad. The trial provided an opportunity to explore participants' views and experiences of 2 weight loss approaches offered in primary care, and participants were purposefully sampled to capture a range of attitudes and experiences. Our findings, however, may not be representative of all overweight people in the United Kingdom who would benefit from weight loss interventions. Indeed, participants in this research were predominantly white British women, and it is important to consider the different needs of men and other ethnic groups, as well as possible cross-cultural variations. Likewise, there may be important differences in the intervention experience based on education, socioeconomic status, and expectations regarding the nature of the prevailing national health care provision, as these differences might well lead to different variants of the explanatory models held about being overweight.

Participants welcomed the offer of weight loss support from their general practitioner. Providing support for weight management outside a medical context by referral to a commercial program, however, fitted better with participants' general explanatory model of being overweight. Our findings further strengthen the evidence of greater weight loss in a commercial program than with standard care $\mathrm{e}^{10,11}$ and support the use of some commercial weight loss programs as part of a range of evidence-based weight loss interventions available in primary care in the United Kingdom. Other health care providers may also wish to consider including interventions outside the traditional medical setting in their service provision. It is likely different groups of people hold variants of the explanatory model that may have subtle, but significant, differences on the effectiveness or acceptability of treatment options. Further research should explore these different models of overweight and also examine patient experience of other weight loss interventions.

To read or post commentaries in response to this article, see it online at http://www.annfammed.org/content/11/3/251.

Key words: obesity; overweight; primary health care; weight loss programs; public-private sector partnerships; qualitative research

Submitted November 22, 2011; submitted revised July 3, 2012; accepted August 8, 2012.

Acknowledgments: We would like to thank the participants and the staff of participating primary care practices for their involvement with the trial and their assistance with data collection. We also thank our collaborators in those institutions from which these participants were drawn.

Funding support: This study was funded by the Medical Research Council [Unit Programme U105960389]. The trial from which participants were recruited was funded by Weight Watchers International through a grant to the Medical Research Council.

\section{References}

1. World Health Organization. Global Health Risks: Mortality and Burden of Disease Attributable to Selected Major Risks. Geneva, Switzerland: World Health Organization; 2009. http://www.who.int/healthinfo/ global_burden_disease/GlobalHealthRisks_report_full.pdf.

2. Franz MJ, VanWormer JJ, Crain AL, et al. Weight-loss outcomes: a systematic review and meta-analysis of weight-loss clinical trials with a minimum 1-year follow-up. J Am Diet Assoc. 2007;107(10):1755-1767.

3. US Department of Health and Human Services. Centers for Medicare and Medicaid Services.Decision Memo for Intensive Behavioral Therapy for Obesity (CAG-00423N). Baltimore, MD: Centers for Medicare and Medicaid Services; 2011. http://www.cms.gov/ medicare-coverage-database/.

4. Counterweight Project Team. Evaluation of the Counterweight Programme for obesity management in primary care: a starting point for continuous improvement. Br J Gen Pract. 2008;58(553):548-554.

5. Nanchahal K, Townsend J, Letley L, Haslam D, Wellings K, Haines A. Weight-management interventions in primary care: a pilot randomised controlled trial. Br J Gen Pract. 2009;59(562):e157-e166.

6. Tsai AG, Wadden TA, Rogers MA, Day SC, Moore RH, Islam BJ. A primary care intervention for weight loss: results of a randomized controlled pilot study. (Silver Spring). 2010;18(8):1614-1618.

7. National Institute for Health and Clinical Excellence (NICE). Obesity: guidance on the prevention, identification, assessment and management of overweight and obesity in adults and children. 2006. http://www.nice.org.uk/CG43.

8. Ahern AL, Olson AD, Aston LM, Jebb SA. Weight Watchers on prescription: an observational study of weight change among adults referred to Weight Watchers by the NHS. BMC Public Health. 2011;11:434.

9. Stubbs RJ, Pallister C, Whybrow S, Avery A, Lavin J. Weight outcomes audit for 34,271 adults referred to a primary care/commercial weight management partnership scheme. Obes Facts. 2011;4(2):113-120.

10. Jebb $S A$, Ahern $A L$, Olson $A D$, et al. Primary care referral to a commercial provider for weight loss treatment versus standard care: a randomised controlled trial. Lancet. 2011;378(9801):1485-1492.

11. Jolly K, Lewis A, Beach J, et al. Comparison of range of commercial or primary care led weight reduction programmes with minimal intervention control for weight loss in obesity: lighten Up randomised controlled trial. BMJ. 2011;343:d6500.

12. Pope C, Ziebland S, Mays N. Qualitative research in health care. Analysing qualitative data. BMJ. 2000;320(7227):114-116.

13. Kleinman A, Eisenberg L, Good B. Culture, illness, and care: clinical lessons from anthropologic and cross-cultural research. Ann Intern Med. 1978;88(2):251-258.

14. Wang SS, Wadden TA, Womble LG, Nonas CA. What consumers want to know about commercial weight-loss programs: a pilot investigation. Obes Res. 2003;11(1):48-53.

15. Tham M, Young D. The role of the General Practitioner in weight management in primary care-a cross sectional study in General Practice. BMC Fam Pract. 2008;9:66.

16. Thomas SL, Hyde J, Karunaratne A, Kausman R, Komesaroff PA. "They all work...when you stick to them": a qualitative investigation of dieting, weight loss, and physical exercise, in obese individuals. Nutr J. 2008;7:34

17. Sturges JE, Hanrahan KJ. Comparing telephone and face-to-face qualitative interviewing: a research note. Qual Res. 2004;4(1):107-118. 